\title{
L'IMAGE DE PIÉTÉ CHRÉTIENNE, OBJET-SUPPORT DE LA CROYANCE ? COMMUNIQUER LA FOI PAR L'IMAGE, DE L'IMPRIMÉ AU NUMÉRIQUE
}

\begin{abstract}
David Douyère ${ }^{1}$
Un certain nombre de sites web chrétiens proposent des images numériques, fixes ou animées, pour soutenir la piété, accompagner la prière des croyants. L'image semble ainsi un rappel à et de la foi, autant qu'une figuration de la présence du Christ, de la Vierge Marie ou des saints. Ces images semblent s'inscrire dans la tradition de l'image de piété catholique. Support de la foi populaire, objet communiquant ordinaire accompagnant les pratiques de piété, les suggérant ou les rappelant, l'image de dévotion catholique, qui s'est largement répandue au XIX ${ }^{\text {ème }}$ siècle, marquant les temps (première communion, ordination, mariage, obsèques) comme les lieux de la vie chrétienne (pèlerinages, sanctuaires), a-t-elle ainsi connu une transmutation numérique, développant, à côté de sa diffusion imprimée, au format $6 \times 9 \mathrm{~cm}$ ou approchant, des formats numériques ? Cet article propose, en s'appuyant sur l'histoire de l'image pieuse et sur une enquête sur les images chrétiennes numériques en ligne, une investigation des figures et des formats de l'image pieuse en ligne pour examiner le rôle de ces supports dans la construction de la croyance, et le rôle des Ntic dans l'évolution des pratiques de dévotion religieuse.
\end{abstract}

1 David Douyère est Maître de conférences de sciences de l'information et de la communication et chercheur au Labsic, Université Paris 13.

Recherches en communication, $\mathrm{n}^{\circ} 38$ (2012). 
La croyance ne naît pas seule. En régime chrétien, et notamment catholique, elle se soutient d'images qui en reconduisent les figures fondatrices ou mythiques, les rendent présentes, incitent à la prière, tissent l'échange entre les membres de cette communauté visible et invisible de croyants et de saints. "L'orant ne prie pas seulement au milieu des choses, mais avec elles ». « De tous les objets que la prière, tel un arbre, tient dans son réseau de gestes, elle fait son dialogue avec Dieu. » L'homme priant, écrit en effet Michel de Certeau, « s'entoure de choses ", "d'objets mis à part, bénis et consacrés, qui épellent son silence et deviennent le langage de ses intentions » (1987, p. 38). L'image de dévotion serait ainsi cet objet-support de la croyance : s'il ne crée pas celle-ci, il en constitue un appui, contribue à sa construction, et en forme un indice. Ainsi, l'image, que l'on dit muette (Bernas, 2006 ; Bœspflug, 2007), dirait là où le croyant ne dit pas, elle dirait des choses à sa place, l'aiderait à (se) dire. L'image de piété « montre » l'objet de la foi ou ceux qui partagent avec le croyant cet objet; elle participe donc de la co-construction de la croyance du chrétien. Elle l'entoure. Elle transmet et co-construit ce que la tradition iconographique et la normalisation ecclésiale (par exemple, Molanus, 1570) ont élaboré d'images de la foi, inscrivant le croyant dans un collectif et un ensemble de pratiques historiquement institués. Cette diffusion connaît aujourd'hui des mutations, par les dispositifs de télécommunication numérique en réseau (Cottin \& Bazin 2003 ; Mayer, 2008 ; Howard, 2011) : l'image de dévotion s'y transforme et anime, s'y diffuse autrement, change de médium mais reste « image » (Belting, 2004), également intérieure et mentale.

La présente recherche, qui s'inscrit dans la perspective plus large d'une investigation des liens entre religion chrétienne et communication (Douyère, 2010, 2011a), entend mener une réflexion sur le rôle l'image de piété chrétienne dans la construction de la croyance, en suivant l'évolution des médiums de l'image de dévotion, de l'imprimé au numérique. Nous souhaitons par conséquent interroger ici les représentations chrétiennes catholiques proposées sur Internet, qui semblent constituer de nouvelles images de piété, et montrer qu'elles représentent un renouvellement ou une prolongation, dans l'accompagnement de la croyance, de l' " image pieuse » catholique traditionnelle. Cette dernière apparaît dès le $\mathrm{XV}^{\mathrm{e}}$ siècle (gravure sur bois), se développe notamment à Anvers, puis s'industrialise et se diffuse très largement, au format $6 \times 9$, à partir du XIX ${ }^{\text {ème }}$ siècle, 
sans se réduire à la forme sulpicienne des éditeurs du quartier de Saint-Sulpice à Paris (Albaric, 1996 ; Hahn, 1984), qui en est la forme la plus connue. Cette généalogie possède à la fois un caractère évident et aberrant : évident car ces images proposées ou envoyées sur Internet sont souvent des images de piété, anciennes, recyclées, ou nouvelles, numérisées ; aberrant car leur mode de production, leur destination, leur nature même et leur usage diffèrent complètement. L'une et l'autre participent toutefois de la pratique « illustrée », se tenant de supports, de la croyance.

Les « petites images » de dévotion imprimées, qui ont accompagné le développement de la foi, notamment populaire, résultent d'une production industrielle, à la différence, à ce jour, des images numériques, qui restent artisanales. Elles participent cependant de la transmission et de la co-construction de la croyance entre le fidèle et son Église, les clercs, et 1'« Esprit » saint, dont les chrétiens posent l'existence. Ces images sont des objets communicationnels, sinon transitionnels, en tant que produits de la propagande chrétienne, outils de mise en ordre morale (Bœspflug, 2007, p. 49 ; Albaric, 1975), instruments de la diffusion d'un message en proximité vers des publics peu lettrés, mais aussi médiateurs possibles d'une communication personnelle avec le divin. Elles portent au recto une scène ou une figure de la foi chrétienne, au verso souvent une prière, et se lient à la vie sacramentelle ecclésiale de celui qui la reçoit, ou la donne. Leur usage confine parfois à la magie quand elles deviennent gris-gris, protègent une étable ou une maison, la chambre d'un enfant, ou, en France, les courriers des premiers facteurs royaux. La « grande conversion numérique » (Doueihi, 2008) et le développement d'une « cybersacralité » (Mayer, 2008) a également touché ces images, soit pour les convertir en un autre support, pour la prière, le souvenir ou la collection, soit pour les renouveler à l'aide d'animations multimédias : gifs animés, powerpoints chrétiens, animations flash religieuses, complètent et renouvellent, au moins techniquement, l'image de piété traditionnelle. Jouent-elles toutefois le même rôle dans l'accompagnement et la construction de la croyance ?

Nous appuyant sur la littérature consacrée à l'imagerie de piété (l'état de l'art est présenté au point 2), nous proposons de considérer des pratiques liées à l'image de dévotion numérique (dans la troisième partie), à partir d'une étude de «l'offre » proposée en ligne et de certaines pratiques qui y sont observables, mais aussi à partir des témoignages 
de chrétiens sur ce type d'images animées. Il nous paraît toutefois important au préalable de rappeler quelques éléments nécessaires à la compréhension de l'image chrétienne, qui a suscité beaucoup d'intérêt et de commentaires.

\section{Quelques précisions sur les images chrétiennes}

À l'instar des travaux d'historiens (par exemple, Christin, 2003), les études en communication n'ont pas négligé la mobilisation de l'image dans la diffusion et la propagande de la foi chrétienne : les essais de Régis Debray $(1992,2001,2003)$ et les travaux des médiologues des Cahiers de médiologie et de Medium, la réflexion de Corinne Abensour (2001, 2000), notamment, ont montré comment l'image a été un vecteur théologique, communicationnel, pour la foi chrétienne et pour l'Église catholique, différenciant une « Église de la parole » d'une « Église du visible » et opposant l'iconophobie protestante et l'iconophilie catholique (Abensour, 2000), opposition parfois contestée (Cottin, 2007). Si les travaux ne manquent pas en philosophie (Mondzain, 1996, 2003, 2007), en histoire de l'art (Belting, 1998, 2007) et en esthétique qui portent notamment sur l'icône et l'art religieux en lien avec la question de la représentation de Dieu (Bœspflug, 2008 ; Bœspflug \& Lossky, 1987), ils font en revanche davantage défaut sur l'imagerie populaire religieuse entendue comme vecteur quotidien et courant de la foi, dans une perspective communicationnelle. La petite imagerie de piété, dite aussi de dévotion, semble en effet intéresser principalement les historiens de l'image populaire, de la piété et de ses objets, régionalement situés, ou les écrivains épris de kitsch religieux nostalgique (Vircondelet, 1988), et, surtout, les amateurs, davantage que les chercheurs en communication. Objet non noble, souvent populaire, industriel (Lerch, 2002), l'imagerie de piété a été souvent décriée sinon rejetée par des traditions spirituelles et esthétiques qui se voulaient plus élaborées (Gamboni, 1999 ; Besançon, 1994 ; Abensour, 2000). Les images de dévotion constituent cependant l'un des objets de proximité de la croyance, insérées dans les missels, ou formant l'image d'un mini-autel de dévotion personnel.

Le christianisme, comme religion de "l'incarnation de Dieu », « fait homme » en Jésus-Christ, a su mobiliser l'image comme vecteur - en accompagnement et en pédagogie de la foi, produisant des signes de l'immatériel objet de la croyance - ou encore l'a dénoncée 
précisément pour sa force substitutive, l'abus de la représentation. Le « voir » et l'image détiennent symboliquement une place centrale dans la foi chrétienne : l'expérience humaine, rachetée par le Salut, ne s'achève-t-elle pas métaphoriquement, pour les Catholiques, dans la Vision béatifique (contemplation de Dieu) semblable à celle des anges (Douyère, 2011c) ? L'ensemble des créatures immatérielles, mais aussi le monde, la nature, le Christ, l'Homme, ne sont-ils pas, dans la théologie médiévale, pensés en termes d'images et de miroir de Dieu, dont la création ne serait qu' une « image »? L'image et le voir possèdent ici un sens théologique qui disent la "réalité » du lien avec Dieu, et ne s'appliquent pas à toute image. Seule l'icône chrétienne orthodoxe, représentation traditionnelle élaborée dans la prière, et fort éloignée de l'icône informatique (pictogramme qui permet le déclenchement d'une action logicielle), produit cette " théophanie » (cette manifestation de Dieu) et suscite, en tant qu'indice de sa présence, vénération (Ouspensky, 1980).

L'image pieuse imprimée, ou numérique, n'est pas l'icône, même si elle peut, aujourd'hui reproduire des icônes, et le fait d'ailleurs abondamment. En effet, l'icône chrétienne orthodoxe (grecque ou russe, mais pas seulement), dogme de la foi, fait partie de la liturgie orthodoxe et relève d'une théologie très spécifique : l'icône, « vision de la Face de Dieu en l'Homme » (Clément, 1993), est liée à l'Incarnation du Christ, Dieu fait homme, et la représente, autant qu'elle porte la grâce divine (Clément, 2010, p. 99). Ceci en fait un instrument de la manifestation de Dieu (Ouspensky, 1980 ; Mondzain, 1996, 2003 ; Sers, 2002 ; Besançon, 1994), et non seulement de la représentation de celui-ci.

Si la place et le sens donnés aux images ont varié suivant les courants spirituels et les époques de la tradition catholique (Abensour, 2000), l'image accompagne la piété et n'appelle pas nécessairement la vénération, elle n'est pas porteuse d'une telle signification théologique.

Depuis les travaux, fondés sur la collection et l'analyse, de Pierre Lessard (1981) au Québec, Jean Pirotte (1987) en Belgique, de Waltraut Hahn $(1984,2007)$ en Allemagne, de Catherine Rosenbaum-Dondaine (1984), de Dominique Lerch $(1982,1992,2002)$ et du père Michel Albaric en France (1975, 1983, 1993, 1996), l'image de piété est désormais l'objet d'une attention qui grandit (Beauducel, 2009 ; 
Cammarano \& Florian, 2009; Oberson, 2001). Elle a toutefois longtemps été stigmatisée comme " imagerie sulpicienne » et a souffert, comme l'imagerie populaire, d'un relatif désintérêt savant, tant des historiens de l'art que des spécialistes de l'art chrétien, compensé par l'intérêt des amateurs et des collectionneurs de « vieux papiers ». La place et la portée des images pieuses est en effet singulièrement absente, tant des travaux sur l'icône et la représentation (Mondzain, 2003, 2007), que sur l'art chrétien (Bœspflug, 2008 ; Bernard, 1999). Est-elle un objet méprisable, signe d'une propagande catholique et d'une piété désuète, cachée dans les missels des grands-mères ? Ne peut-elle être revisitée aujourd'hui que sous le biais du kitsch, en art ou par la mode ? N'a-t-elle pas cependant joué un rôle central dans la diffusion de la foi chrétienne, et l'activation ou le maintien de la croyance?

\section{La construction de la croyance par l'image de piété imprimée}

Si, " avant que d'être commune, la croyance a dû être communiquée » (Jacques, 1979, p. 266 ; Jacques, 2005), les images de dévotion imprimées ont fortement participé de la communication du système de croyance chrétien, au côté de nombreux autres dispositifs (sacrements et liturgie, architecture, prédication, catéchèse, confession, accompagnement spirituel...). Pour l'anthropologue du religieux Albert Piette, l'état de croyance, ou plutôt l'acte de croire peut être défini comme la « rencontre ponctualisée entre une représentation semi-propositionnelle ambiante et une disposition affective ou un processus intellectuel propre à l'individu présent en situation » (Piette, 2003, p. 68). L'image, comme le texte et la parole religieuse qui s'imbriquent (Cottin, 2007), concourt à la constitution de cette " représentation semi-propositionnelle ambiante ». Force d'é-motion (« [...] l'image est é-motion. Plus que l'idée, elle met les foules en mouvement ", Debray, 1992, p. 97), son pouvoir spécifique est sans doute de pouvoir jouer sur l'affect, ce dont les images de piété chrétiennes ne se sont pas privées (Albaric, 1975 ; Pirotte, 1987).

La «petite image de piété» ou « de dévotion», est en effet inséparable de la spiritualité catholique, au point qu'elle a pu être envisagée comme pouvant servir de base à une « histoire du sentiment religieux » par les objets (Albaric, apud Lerch, 2007). L'image de piété semble apparaître au XIV ${ }^{\text {ème }}$ siècle en Europe dans un contexte et une perspective, selon 
Menozi (1991, p. 127), d'essor de la piété individuelle chez les laïcs, et d'un passage de la pratique dévote de l'Eglise à l'habitation privée (Menozi, 1991, p. 128). Elle connaît plusieurs fonctions. Trace, souvent personnalisée, de façon imprimée ou manuscrite, d'un sacrement constituant un événement dans la vie personnelle du croyant (baptême, " première communion », ordination sacerdotale, décès), ou d'un évènement spirituel (année mariale, pèlerinage...) ; évocation d'un saint ou d'une figure majeure de l'Église (les papes Paul VI, Jean-Paul II, Benoît XVI) ; émanant d'une congrégation religieuse, d'une société de piété (rosaire, adoration eucharistique) ; objet que l'on transporte, manipule, perd et retrouve, l'image de piété s'achète ou se reçoit, se donne en signe d'amitié spirituelle (Albaric, 1996) et se conserve, le plus souvent entre les pages d'un missel (lectures de l'office ou de la messe du jour), consulté ou non. Proche de l'image et de la fonction des catéchismes illustrés (Saint-Martin, 2000), elle a pu assurer une transmission des « mystères » chrétiens, et diffuser les fondamentaux de la foi ou de la vie sacramentelle.

L'image pieuse imprimée (elle peut aller jusqu'à comporter plusieurs volets, en dépliants), ornée ou non de « dentelle » de papier (on parle alors de " canivet », Taveneaux, 1992), pourvue parfois d'un morceau de « relique » ou d'un fragment d'objet ayant un lien quelconque avec un saint, porte en son recto un dessin ou une gravure, une photographie, une reproduction d'un tableau ou d'une sculpture, une figure " évocatrice » de la foi, et en son verso un texte imprimé (le plus souvent dans un second temps), extrait d'évangile, parole d'un saint, prière, mémoire d'un événement. Elle intrique donc étroitement, par ses inscriptions (réalisées souvent par deux imprimeurs, au recto, pour l'image standard, et au verso, pour la personnalisation) ou son mode d'usage, la vie personnelle et la foi. Elle «fait lien », en un sens, entre l'un et l'autre.

L'image de dévotion participe de la construction de la croyance, en un premier sens, en ce qu'elle peut être regardée pour prier, et accompagne la prière. En effet, elle comporte le plus souvent à son verso une prière, ou les indications d'un mode de prière (litanies, prière au cœur de Jésus, etc.), tandis qu'au recto, la figure du Christ ou de la Vierge, plus rarement de Dieu le Père ou de la Trinité, en rappelle l'objet. L'image de piété vient donc accompagner la prière, solitaire, contemplative ou de demande (prière que le croyant catholique adresse 
à un saint, au Christ ou à la Vierge pour obtenir quelque chose dans sa propre vie, matérielle ou spirituelle). L'image de dévotion est donc en un sens une « mémoire», au double sens, que, médiation, elle fait signe vers l'objet, ou un médiateur et témoin de la foi, et qu'elle fait souvent mémoire d'un événement personnel. Objet mobile (et léger), petit, elle circule et fait circuler le sentiment, la prière, la subjectivité croyante. Objet du « banal » chrétien catholique, elle fait partie d'un ensemble de dispositifs, objets, accompagnant, jusqu'à la seconde moitié du $\mathrm{XX}^{\mathrm{e}}$ siècle, la pratique chrétienne, parmi les médailles, crucifix, statuettes, etc., et partage avec ces derniers une « imagerie » commune.

Mais l'image de dévotion participe aussi de la construction de la croyance, en un deuxième sens, où la croyance religieuse voisine la croyance magique : l'image est alors perçue comme porteuse de pouvoirs et, apotropaïque, se fait « image de préservation ». Ainsi Lessard (1981, p. 39) montre-t-il, dans son ouvrage sur les images pieuses au Québec, que l'image de piété était « utilisée comme talisman contre les incendies et la foudre ", " contres les ravages de la petite vermine », qu'elle était épinglée dans les tiroirs ou dans la cave pour protéger les aliments. Ainsi les images « étaient [...] destinées [...] à protéger les gens et les biens matériels contre les multiples risques de la vie quotidienne : maladies, accidents, incendies, etc. ». « Dans la maison, épinglée sur un mur ou dans une armoire, l'image de petit format n'était donc en fait qu'un objet immobile (une partie sacrée du décor) voué à la protection immédiate des occupants et des lieux ». Cet autre aspect de la croyance, qui gêne la rationalisation ecclésiale de la foi et se trouve remisé du côté de la superstition, n'en est pas moins une autre dimension de l'image de dévotion qui ouvre un lien, y compris matériel, avec le spirituel, dans une dimension proche, notamment, de la prière de demande, par lequel un chrétien demande à une figure spirituelle (un saint, le Christ, la Vierge Marie) d'être exaucé. Comme l'écrit Lessard (1981, p. 39), une double construction de la croyance s'effectue via l'image de piété : «L'image de petit format faisait donc autrefois partie d'un ensemble d'artefacts religieux destinés, d'une part, à édifier, à instruire et à sensibiliser les fidèles et, d'autre part, à les protéger en servant de talismans, de porte-bonheur ». Qu'en est-il de l'image de piété numérique? 


\section{Croyance et image de piété chrétienne sur les réseaux numériques}

L'image religieuse chrétienne semble aujourd'hui profuse sur le Web : envoyée par mail, diffusée de façon dite « virale » sur les sites de réseaux sociaux numériques, présentée et proposée sur des sites Web, elle est accessible suivant des modes de diffusion très différents, qui distinguent ou associent le résidentiel (mise en ligne sur un serveur) et l'envoi (courriel, réseau social). Ses supports et ses formats numériques sont également très variables : image fixe au format jpeg proposée sur un site, images fixes dans un fichier $p d f$ ou une présentation de type powerpoint, animée (format gif, flash) ou non, vidéo. Elle emprunte les formes que le multimédia propose en ligne. Cette profusion, ces différents modes de circulation et de diffusion ou d'acheminement, cette diversité des formats posent un problème à la fois d'accès et de recensement, de représentativité même du corpus sélectionné.

Nous avons procédé, pour cette investigation exploratoire, par recherche de mots-clefs sous un moteur de recherche courant et actuellement dominant sur le marché, Google, en sollicitant la fonction image de celui-ci. Les mots-clefs utilisés sont : Jésus, Jésus-Christ, Vierge Marie, Marie, Pape, images pieuses, images de piété, powerpoint chrétien, powerpoint Jésus, powerpoint Jésus-Christ, powerpoint Marie. Cette recherche, qui a généré une navigation suivant l'hypertexte, donc les ramifications ouvertes par l'intérêt des liens, introduisant une relative dérégulation autant que favorisant sa propension heuristique, s'est trouvée complétée par une sollicitation de mails auprès d'un échantillon non représentatif de personnes, afin de d'avoir quelques traces d'envoi. Il s'agissait avant tout, dans la perspective de cette étude, de cerner l'offre en ligne d'images pieuses numériques.

Le corpus constitué comporte une vingtaine de sites $^{1}$. Il est possible de les classer en quatre grandes catégories : sites personnels (amateurs

1 Parmi les principaux sites consultés lors de l'enquête (2010-2011), on peut citer http://la.mesange.chez-alice.fr/images\%20pieuses.htm, http://www.spiritualitechretienne.com/, http://jesuschrist.e-monsite.com/rubrique, donner-une-rosea-jesus,302830.html, http://www.la-boutique-des-chretiens.com, http://www. blog-catholique.com, http://lieudepriere.free.fr/litanies_sacre_coeur.htm, http:// jesuschrist.unblog.fr/2008/12/02/bonjour-tout-le-monde/comment-page-2/, http:// 
ou croyants), sites commerciaux, sites de communautés religieuses, sites de paroisses ou d'évêchés. Les supports recensés sont les suivants : images jpeg ou gif, accompagnés ou non de fichiers audio, présentation powerpoint, animations flashs.

L'entrée par mots clefs à partir d'un moteur de recherche fait accéder à un grand nombre d'images qui se trouvent dans des contextes numériques fort distincts, de la collection et du commerce au musée, en passant par la dimension religieuse. L'image y est tantôt donnée pour ce qu'elle représente, ou comme une représentation d'une autre image, imprimée, dont il s'agit de décrire ou de favoriser la collection. Suivant le contexte, la fonction de collection, la fonction esthétique ou de connaissance et la fonction spirituelle sont mises ou non en avant, les trois pouvant en réalité plus ou moins s'exercer sur chaque image. On voit donc que la dimension d'archive joue en un double sens : archive culturelle d'une histoire des images ou de la piété, et archive spirituelle, au sens de restitution d'un état passé de la piété, pour le cas de sites catholiques traditionalistes, nombreux à mobiliser ces images, souvent spirituellement et iconographiquement datées.

Les formats font des images religieuses des réalités plus ou moins complexes. À la seule figuration d'un sujet d'une image fixe répond la multiplicité des images d'une présentation powerpoint (du nom du logiciel de PréAO ${ }^{1}$ de Microsoft) assemblant et combinant, avec animation ou non, plusieurs images. Si les images fixes ${ }^{2}$ sont les plus nombreuses et l'emportent sur les gifs animés (par exemple, un cercle clignote autour de Jésus et Marie, ou l'eau derrière le Christ s'anime), formant une « cybercarte 3 » et sur les associations image-musique ${ }^{4}$ et les animations flash $^{5}$, nous souhaiterions ici focaliser notre attention sur un type particulier d'image de piété, scénarisé, l'assemblage animé et interactif d'images fixes constitué par la présentation powerpoint

laflammedamour.tripod.com/lapriere.html, http://divineprovidence.e-monsite.com cartes.html, http://www.mariedenazareth.com/3776.0.html?\&L=0, http://www. sm2m.ca.

1 Présentation assistée par ordinateur.

2 Elles apparaissent parfois sous le format de "cartes postales virtuelles", telles sur http://www.spiritualite-chretienne.com/e-cards/cartes.html.

3 http://divineprovidence.e-monsite.com/cartes.html.

4 http://jesuschrist.unblog.fr/2008/12/02/bonjour-tout-le-monde/comment-page-2.

$5 \mathrm{http}: / /$ www.mariedenazareth.com/3776.0.html?\&L=0. 
de méditation, que nous pourrions qualifier par voie de raccourci de «powerpoint chrétien ».

La PréAO de méditation, ou « diaporama chrétien », requiert en effet comme dispositif scriptovisuel et sonore, une attention spécifique car elle constitue certainement une " innovation » liée au support numérique. Support multimédia qui peut être dit interactif en ce qu'il requiert le clic de l'utilisateur (qui n'en infléchit toutefois pas le déroulement, ce qui ne saurait toutefois suffire à définirl'interactivité), les powerpoints chrétiens, tels ceux de l'Abbaye bénédictine Sainte-Marie des Deux-Montagnes ${ }^{1}$ au Québec (Douyère, 2011b), introduisent une dimension multimédia et, surtout, un contrôle du temps dans la méditation. Constitués d'images religieuses ${ }^{2}$ en un support logiciel préconstruit, augmentées de textes et de musique, réalisés par une sœur peintre d'icônes, ces powerpoints se présentent comme un dispositif (automatisé, donc) d'accompagnement de la prière : ils sont un support de communication, éventuellement pédagogique ${ }^{3}$. Il s'agit aussi, selon leurs conceptrices, d'un outil de mise en présence de Dieu, sous la figure du Christ, de la Vierge Marie ou des saints. Une attention particulière est portée à la temporalité spécifique de la méditation. Le document avertit en effet : «Ce diaporama est une prière, prendre son temps pour contempler les images et écouter le chant d'adoration ${ }^{4} \gg$. Une sorte de liturgie (numérique), analogue à celle d'un office religieux, est mise en place. On a ici affaire à un usage non managérial du powerpoint, étudié par ailleurs par Jeanneret (et al., 2007), d'Huy (2007) ou décrit par Frommer (2010) comme simplificateur de la pensée. Cependant, que des parallèles avec le powerpoint managérial, sur le plan de l'adhésion et de la croyance, sinon bien sûr de la conviction emportée par le visuel, pourraient être établis.

Si la construction d'une temporalité spécifique de la prière, avec construction d'un cadre, n'est pas une nouveauté (les images de piété sous forme de dépliants, développées par exemple à la fin du XIX ${ }^{\text {ème }}$

$1 \mathrm{http}: / /$ www.sm2m.ca.

2 «Les œuvres d'art sont celles qu'on regarde et qu'on aime au monastère », déclare une sœur en entretien (cf. infra). Elles sont trouvées parmi les livres d'art chrétien de la bibliothèque, puis scannées.

3 Rorate coeli, Cieux_repandez_votre_rosee.pps

4 « Nuit_au_tombeau.pps », http://www.sm2m.ca/popup.asp?s=4\&ss=5\&sss=1. 
siècle, le faisaient également), et fait partie des techniques d'oraison et de médiation chrétiennes, très variées, l'application multimédia s'efforce ici de construire un univers méditatif par la présence conjointe de la musique religieuse et de l'image, et d'un temps lent de l'animation. Le dispositif multimédia intervient donc ici dans une co-construction de la médiation orante, ou plutôt, il intègre des éléments d'une pensée préalable de l'oraison, conçue par les sœurs de l'Abbaye Sainte-Marie des Deux-Montagnes au Québec. Une anticipation de la méditation est donc à l'œuvre dans ce type d'objet, semblable, après tout, à celle qui a dû exister dans la conception d'une image de piété destinée à l'oraison. On pourrait à ce propos parler de "PriAO", de «prière assistée par ordinateur " (Douyère, 2011b), pour désigner ce dispositif où l'application logicielle, comme autrefois l'image imprimée augmentée de textes de médiation, fait entrer dans une temporalité orante, et accompagne la prière. Nous sommes en tout cas en présence d'une image-action (Barboza, \& Weissberg, 2006) tant par ce qu'elle est que par ce qu'elle fait faire.

Les témoignages recueillis par les sœurs bénédictines de l'abbaye Sainte-Marie des Deux Monts ${ }^{1}$, et dont elles ont bien voulu nous communiquer une partie, semblent indiquer un lien fort entre l'image, animée, mise en scène, en musique et en prière, et la croyance. L'un d'entre eux évoque « les images à couper le souffle» (témoignage 4); un autre le fait de « faire prier sur de la beauté » (témoignage 6). C'est d'une entrée profonde dans la prière, dont témoigne un autre :

Je ne sais pas si ce mail arrivera quelque part mais l'émotion est si forte que je dois la partager. Peu importe avec qui, peu importe où. Je reçois en ce vendredi Saint un diaporama qui porte cette adresse mail. « Jésus »... « Jésus »... « Jésus »... entre le chant, la méditation qui l'accompagne je me sens si près de Lui que mes yeux se sont remplis de larmes. Je le regarde encore et encore... je Le regarde encore et encore. Je n'aurai[s] su trouver moyen plus fort de Le rejoindre dans sa Passion... Merci. (témoignage 10).

Ici, l'image animée semble accompagner la foi, sinon la croyance, et la transformer en émotion et sentiment de présence, au moment de

1 Plus de 620 au 7 février 2011. 
la célébration de Pâques. L'expérience orante se co-construit avec le diaporama, qui évoque une présence, qui lui préexiste.

La dimension mystagogique des images apparaît dans un autre écho donné de la consultation de ces animations ( «Les images [...] nous invitent à participer au Mystère », témoignage 12). "Les images sont d'une beauté saisissante », écrit un autre correspondant (témoignage 14). Plusieurs relient ainsi cette expérience de la consultation des diaporamas à l'expérience de la prière : " Il est un petit chef d'œuvre qui nous met d'emblée en " présence du Mystère » et nous " relie au divin » » (témoignage 18). Une pratique nouvelle de prière individuelle face à des images de piété, renouvelée par l'animation et l'accompagnement sonore que permet le support numérique, semble ainsi se créer. L'image semble ainsi liée à l'exercice d'une pratique concrète de la croyance.

\section{Conclusion : mutations de l'image de piété traditionnelle et co-construction de la croyance}

L'image de dévotion est donc l'un de ces objets, de papier imprimé ou numérique, qui accompagne la construction de la croyance, et a constitué en tant que tel un des éléments du matériel de la "propagande » chrétienne, éveillant et permettant des pratiques de dévotion, dites « populaires »-encore que rien n'atteste qu'elles n'aient pas été aussi le fait des lettrés -, et reste attaché aujourd'hui à des pratiques chrétiennes plutôt traditionalistes. L'image de piété n'est cependant pas au centre des pratiques de foi chrétienne (comme le sont au contraire la prière, la "pratique de la charité », le partage communautaire et les sacrements, comme l'eucharistie), et peut ne pas trouver sa place dans le cadre d'une ethnographie rapprochée des pratiques chrétiennes (comme par exemple le souligne Piette, 1999).

Dans l'accompagnement du développement des croyances, des mutations sont donc observables quant aux supports de l'image de piété. L'image de piété imprimée, telle qu'elle se développe dans l'Église catholique en Europe à partir du XV ème siècle, et l'image de piété numérique en réseau diffèrent tant dans leur conception que dans leur mode de production industrielle, et aussi bien dans leur usage. L'une et l'autre sont bel et bien cependant des modes de circulation des images (en réseau, isolément, de main à main), qui accompagnent la foi, et de l'image dans l'image, attestant d'une intericonicité, par 
les effets de reproduction, d'inspiration et de copie (Beauducel, 2009). Elles peuvent l'une comme l'autre s'inscrire dans une perspective contemplative, orante et relationnelle (donner à, remettre à, envoyer à une image), dont seule l'étude de l'usage, ou de l'inscription dans une pratique spirituelle peut donner véritablement le sens et la portée.

L'une et l'autre image ont enfin comme "but » la génération d'affects, voire de sentiments, et la mise en mouvement intérieure dans la perspective d'une coloration spécifique de l'éprouvé spirituel. Plusieurs auteurs ont ainsi souligné cette mise en mouvement émotionnel suscitée par l'image religieuse : "L'image a pour spécificité d'émouvoir et donc de mouvoir » (Mondzain, 2003, p. 19, p. 27). L'une et l'autre, enfin, sont liées à des textes, qui à la fois les font naître et les accompagnent, et dont elles figurent ou construisent la réalité. À ce titre, l'image de piété, imprimée ou numérique, contribue à « communiquer » la foi.

Si l'image de piété traditionnelle constituait une trace d'un événement personnel et sacramentel (baptême, première communion, ordination, mariage, obsèques) ou spirituel (pèlerinage), et s'échangeait, dès le $\mathrm{XVIII}^{\mathrm{e}}$ siècle, comme trace d'amitié spirituelle, elle est liée à l'imprimé en ce qu'elle s'insère entre les pages de celui-ci, en marque les pages de façon personnelle. Elle contribue, dès lors, autant à un lien spirituel (avec une personne, ou avec l'objet de la croyance) qu'à une personnalisation du livre (missel, livre pieux). Sa dimension communicationnelle, en tant qu'elle évoque l'Évangile et le Christ, intrique le spirituel et le personnel, elle rend présents l'un et l'autre conjointement. L'image fait tourner le regard vers l'objet intérieur de la foi. Une dimension «magique » de protection peut lui être implicitement conférée par le croyant; elle poursuit alors la tradition des « images de préservation » et des " images de protection » anciennes, semblable à un "gri-gri » chrétien, comme certaines statuettes et médailles. L'image de piété numérique, davantage objet apostolique, de diffusion, de propagation de la foi, ne reçoit pas, le plus souvent, d'inscription personnelle et ne se trouve pas liée à un événement sacramentel ou personnel. Si la nature du support, mobile, et réticulaire, ne permet pas qu'elle se " glisse » entre les pages d'un document, on voit bien également qu'elle peut accompagner les documents numériques, les voisiner, et qu'elle s'insère dans l'espace personnel de consultation des documents numériques. Rien n'atteste qu'elle soit fréquemment utilisée à des fins de protection d'un terminal numérique (ordinateur, téléphone) 
ou de son usager. La conversion d'un support à l'autre est donc toute relative : s'il semble y avoir prolongement de forme, la matérialité de l'image de piété imprimée, qui avait une fonction précise comme objet d'accompagnement de la foi, disparaît, tandis que le numérique correspond à de nouvelles modalités, liées aux réseaux numériques, de diffusion de la croyance, sinon d'exercice de la piété chrétienne, dans le cadre de la " nouvelle évangélisation » de ce que le pape Benoît XVI (2010) nomme « le continent numérique ».

Il reste que, liées à la croyance, accompagnement éventuel de la foi, ces images sont, en régime chrétien, vouées à disparaître et ne sont que des béquilles, temporaires, de la foi : " La différence entre voyant et vu, écrit l'historien de la mystique Michel de Certeau dans Extase blanche (1987, p. 309), ne tient plus si aucun secret ne met le voyant à distance de ce qu'il voit, si aucune obscurité ne lui sert de refuge d'où constituer devant lui une scène, s'il n'y a plus de nuit dont se détache une représentation. Voilà ce que serait l'éblouissement de la fin : une absorption des objets et des sujets dans l'acte de voir. [...] Rien de caché, et donc rien de visible. » La fin des images.

\section{Références}

Abensour, C. (2001). Les modalités paradoxales de la présence chrétienne sur Internet. Dans : Émergence et continuité dans les recherches en information et communication, Actes du 12e congrès national de la Sfsic (pp. 105-110). Paris : Sfsic.

Abensour, C. (2000). La place de l'image dans la communication religieuse : Église du visible, Église de la parole. Dissertation doctorale, Université Paris 13.

Albaric, M. (1996). Le commerce des objets religieux dans le quartier Saint-Sulpice. Dans Collectif, De Pierre et de cour, l'église Saint-Sulpice, 350 ans d'histoire. (pp.131-158). Paris : Le Cerf.

Albaric, M. (1993). Saint Bernard dans l'imagerie de piété des XIX ${ }^{\text {ème }}$ et XX ${ }^{\text {ème }}$ siècles. Dans P. Arabeyre, J. Berlioz, P. Poirrier (Éd.), Vies et légendes de St Bernard de 
Clairvaux, création, diffusion, réception (XIIe-XXe s.). (pp. 371-384). Saint-Nicolas lès Cîteaux : Commentarii Cisterciences.

Albaric, M. (1983). Les images religieuses, une polysémie qui exige l'informatique. Le Vieux Papier, 30(287), 12-16.

Albaric, M. (1975). Jésus dans l'imagerie pieuse de 1860 à 1960. Jésus, 8, 29-36.

Barboza, P., \& Weissberg, J.-L. (2006). "L'image actée », Scénarisations numériques. Paris : L'Harmattan.

Beauducel, C. (2009). L'imagerie populaire en Bretagne au XVII ème et XIX Xème siècle. Rennes : Presses universitaires de Rennes.

Belting, H. (2007). La vraie image : croire aux images? Paris : Gallimard.

Belting, H. (2004). Pour une anthropologie des images. Paris : Gallimard.

Belting, H. (1998). Image et culte : une histoire de l'image avant l'époque de l'art. Paris : Le Cerf.

Benoît XVI. (2010). Le prêtre et la pastorale dans le monde numérique: les nouveaux médias au service de la Parole. Message pour la 44è journée mondiale des communications sociales. Cité du Vatican : Conseil Pontifical pour les Communications Sociales.

Bernas, S. (2006). La croyance dans l'image. Paris : L'Harmattan.

Besançon, A. (1994). L'image interdite. Une histoire intellectuelle de l'iconoclasme. Paris : Fayard.

Bernard, J. (1999). Qu'y a-t-il dans une « crise »? « Problèmes » parallèles dans les mouvements spirituels et les images religieuses de la fin du XIX ${ }^{\text {ème }}$ en France. Dans O. Christin, \& D. Gamboni (Éd.), Crises de l'image religieuse, de Nicée II à Vatican II. (pp. 213-238). Paris : MSH.

Bœspflug, F. (2008). Dieu et ses images, Une histoire de l'Éternel dans l'art. Paris : Bayard.

Bœspflug, F. (2007). Sens et non-sens des images au regard de la parole. Lumière \& vie, 275, 43-57.

Bœspflug, F., \& Lossky, N. (Éd.). (1987). Nicée II, 787-1987, Douze siècles d'images religieuses. Paris : Le Cerf.

Cammarano, F., \& Florian, A. (2009). Santini e Storia di un Editore parigino. Maison Bouasse-Lebel [Images de piété et histoire d'un éditeur parisien]. Marene : Astegiano.

Caro Dambreville, S. (2008). Le corps divin entre représentation " imagée » et « langagière ». Dans Corps humains, corps divins, Paris : France. Disponible à : http://halshs.archives-ouvertes.fr/docs/00/45/29/57/PDF/Representation_Divin_ Caro.pdf.

Certeau, M. de (2003 [1987]). La Faiblesse de croire. Paris : Le Seuil.

Christin, O. (2003). Les Yeux pour le croire. Les Dix Commandements en images, $X V^{\text {ème}}$-XVII ${ }^{\text {ème }}$ siècle. Paris : Le Seuil.

Clément, O. (2010). L'Église orthodoxe. Paris : Presses universitaires de France.

Clément, O. (1993). Un art un livre, un appel. Dans M. Zibawi (Éd.), L'icône, sens et histoire. (pp. 5-8). Paris : DDB.

Cottin, J. (2007). Le Christianisme libérateur de l'image. Lumière \& Vie, 275, 29-37.

Cottin, J., \& Bazin, J.-N. (2003). Vers un Christianisme virtuel ? Enjeux et défis d'Internet. Genève : Labor et Fides.

Debray, R. (2003). Le Feu sacré, fonctions du religieux. Paris : Fayard.

Debray, R. (2001). Dieu, un itinéraire. Paris : Odile Jacob. 
Debray, R. (1992). Vie et mort de l'image, Une histoire du regard en Occident. Paris : Gallimard.

Doueihi, M. (2008). La grande conversion numérique. Paris : Le Seuil.

Douyère, D. (2011a). De l'usage chrétien des médias à une théologie de la communication : le père Émile Gabel. Le Temps des Médias, 17, 64-72.

Douyère, D. (2011b). La prière assistée par ordinateur. Médium, 27, 140-154.

Douyère, D. (2011c). Communication : on Being an Angel, Incarnation and Angelic Communication in Medieval Christian Theology. Modern Reflections, Starting with Thomas Aquinas's Summa Theologica. Communication and Critical / Cultural Studies, 8(2), 188-193.

Douyère, D. (2010). Communication et religion chrétienne catholique. Cahiers de la Sfsic, 5, 17-18.

Dunand, F., \& Bœspflug, F. (Éd.). (2002). Voir les dieux, voir Dieu. Strasbourg : Presses universitaires de Strasbourg.

Evdokimov, P. (1970). L'Art de l'icône, théologie de la beauté. Paris : Desclée.

Frommer, F. (2010). La pensée Powerpoint. Paris : La Découverte.

Gamboni, D. (1999). De « Saint-Sulpice » à l'« art sacré », qualification et disqualification dans le procès de modernisation de l'art d'église en France (18901960). Dans O. Christin, \& D. Gamboni (Éd.), Crises de l'image religieuse, de Nicée II à Vatican II. (pp. 239-261). Paris : MS.

Hahn, W. (2007). Un objet religieux et sa pratique. Le chemin de croix portatif aux XIX $X^{\text {ème }}$ et XX'ime siècles. Paris : Le Cerf.

Hahn, W. (1984). Saint-Sulpice une die devotionale bilderindustrie des 19. Jahrhunderts in Frankreich. Mémoire, Université Julius Maximilians, Würtzburg, faculté de philosophie.

Howard, R. G. (2011). Digital Jesus: The Making of a New Christian Fundamentalist Community on the Internet. New York: NY University Press.

Huy, P. d' (2007). PowerPoint, la rhétorique universelle. Medium, 11, 12-25.

Jacques, F. (2005). La croyance, le savoir et la foi. Paris : Presses universitaires de France.

Jacques, F. (1979). « Croyance commune, croyance communiquée ». Dialectica 33, 3-4, 263-281.

Jeanneret, Y., Hamard, J., \& Tardy, C. (2007). L'empreinte sociale d'un outil d'écriture : PowerPoint chez les consultants. Dans C. Tardy \& Y., Jeanneret (Éd.), L'écriture des médias informatisés : espaces de pratiques. (pp. 141-171). Paris : Hermès SciencesLavoisier.

Lambert, F. (2009). Images, langages et médias : essai pour une sémiotique de la croyance. Dans R. le Champion \& C. Leteinturier (Éd.), Médias Information et communication. (pp. 251-262). Paris : Ellipses.

Lejeune, L., \& Neuberg, A. (1990). Imagiers de paradis. Images de piété populaire du $X V^{\text {ème }}$ au XXème siècle. Bastogne : Musée en Piconrue / Crédit communal.

Lerch, D. (2007). Le sentiment religieux, objet d'étude scientifique ? Après l'histoire littéraire, pour une histoire des objets religieux ? Dans W. Hahn, Un objet religieux et sa pratique, le chemin de croix "portatif» aux XIXe et XXe siècles en France. (Trad. fr. de L. Knepfler \& D. Lerch) (pp. 11-26). Paris : Le Cerf.

Lerch, D. (2002). En quoi l'imagerie populaire a-t-elle été une industrie culturelle ? Quelques propositions. Dans Collectif, Histoire des industries culturelles. (pp. 321339). Paris : Association pour le développement de l'histoire économique. 
Lerch, D. (1992). Imagerie populaire en Alsace et dans l'Est de la France. Nancy : Presses universitaires de Nancy.

Lerch, D. (1982). Imagerie et société. L'imagerie Wentzel de Wissembourg au XIXème siècle. Strasbourg : Istra.

Lessard, P. (1981). Les petites images dévotes. Leur utilisation traditionnelle au Québec. Québec : Presses de l'Université Laval.

Mayer, J.-F. (2008). Internet et religion. Gollion (CH) : Infolio.

Menozi, D. (1991). Les Images, L'Eglise et les arts visuels. Paris : Le Cerf.

Molanus. (1996 [1570, 1594]). Traité des saintes images. Paris : Le Cerf.

Mondzain, M.-J. (2007). Homo Spectator. Paris : Bayard.

Mondzain, M.-J. (2003). Le Commerce des regards. Paris : Le Seuil.

Mondzain, M.-J.(1996), Image, icône, économie, Les sources byzantines de l'imaginaire contemporain. Paris : Le Seuil.

Oberson, E. (2001). La Grâce d'être humain : un savoir du cæeur. Essai de dialogue avec le corpus d'images de piété saint-sulpiciennes de la maison DesgodetsLorthioir. Dissertation doctorale, Institut catholique, Faculté de théologie et de sciences religieuses.

Ouspensky, L. (1980). La Théologie de l'icône dans l'Église orthodoxe. Paris : Le Cerf.

Piette, A. (2003). Le fait religieux, une théorie de la religion ordinaire. Paris : Economica.

Piette, A. (1999). La religion de près, l'activité religieuse en train de se faire. Paris : Métailié.

Pirotte, J. (1987). Images des vivants et des morts, La vision du monde propagée par l'imagerie de dévotion dans le Namurois, 1840-1965. Louvain-la-Neuve \& Bruxelles : Nauwelaerts.

Rosenbaum-Dondaine, C. (1984). L'Image de piété en France, 1814-1914. Paris : Musée galerie de la Seita.

Saint-Martin, I. (2000). "Catéchisme en images », une pédagogie par le sensible ? Archives de sciences sociales des religions, 111. Disponible à : http://assr.revues. org/20223.

Sers, P. (2002). Icônes et saintes images. La représentation de la transcendance. Paris : Les Belles Lettres.

Taveneaux, E. (1992). La Piété en dentelles, Les images de dévotion et leurs dentelles 1830-1910. Nancy: Presses universitaires de Nancy.

Vircondelet, A. (1988). Le Monde merveilleux des images pieuses. Paris : Hermé. 